\title{
Incarcerated pericecal hernia: a rare form of the internal hernia
}

\author{
Sung II Kang \\ Department of Surgery, Yeungnam University College of Medicine, Daegu, Korea
}

\begin{abstract}
A pericecal hernia is a rare form of an internal hernia, which has been described in several case reports. We present a case of a 32-year-old woman who complained of vague abdominal pain a day prior to admission. Abdominal computed tomography revealed the presence of a pericecal hernia without bowel ischemia. The patient underwent manual hernia reduction and was discharged without complications. We describe this case in detail and provide a review of the pertinent literature.
\end{abstract}

Keywords: Cecum; Hernia; Intestinal obstruction

\section{INTRODUCTION}

An internal hernia is defined as a bowel protrusion into a congenital or acquired visceral hiatus that can cause intestinal obstruction [1-2]. Pericecal hernia is a rare form of an internal hernia that has been described in several case reports globally including a case in Korea [3]. We report another case of a pericecal hernia in Korea and present a literature review.

\section{CASE}

A 32-year-old woman was admitted to our hospital with complaints of vague abdominal pain a day prior to presentation. She reported having undergone conversion of laparoscopic to open abdominal surgery secondary to symptoms misdiagnosed as pan-peritonitis at another hospital a year ago. She revealed that she underwent only exploratory laparotomy without bowel or other organ resection during the pre-

Received: April 20, 2018, Revised: May 8, 2018

Accepted: May 18, 2018

Corresponding Author: Sung II Kang, Department of Surgery, Yeungnam University College of Medicine, 170, Hyeonchung-ro, Nam-gu, Daegu 42415, Korea Tel: +82-53-620-3580, Fax: +82-31-787-4055

E-mail: sungiry@naver.com vious surgery. There was no other history of surgery and medicine. Upon admission, her body mass index was $20.2 \mathrm{~kg} / \mathrm{m}^{2}$, and her vital signs were stable. Palpation of the abdomen revealed direct tenderness in the periumbilical area without rigidity, distension, and/or a mass-like lesion. Blood tests revealed that her white blood cell count had increased to $11,900 / \mu \mathrm{L}$, although other parameters were within reference ranges. An abdominal X-ray revealed a nonspecific bowel gas pattern. Abdominopelvic computed tomography (CT) demonstrated an abnormal location of the cecum, which was positioned towards the pelvis, and an ileal segment showed kinking of the mesenteric vessels located below and at the right lateral aspect of the ascending colon (Fig. 1). An emergency operation was performed because of persistent abdominal pain and the risk of bowel strangulation, although no bowel dilatation and/or abnormal bowel perfusion were observed on CT.

The patient initially underwent a single-port exploratory laparoscopy via the previous operative site. Laparoscopically, after we performed adhesiolysis, an ileal segment was observed on the lateral aspect of the ascending colon, and herniation was observed below the cecum (Fig. 2). Careful reduction was performed using a laparoscopic bowel grasper; however, marginal mesenteric tearing of the ileum caused hemorrhage, necessitating conversion of the laparoscopic procedure into an open abdominal surgery. After manual reduction of the herni

Copyright $($ C) 2018 Yeungnam University College of Medicine

This is an Open Access article distributed under the terms of the Creative Commons Attribution Non-Commercial License (http://creativecommons.org/licenses/by-nc/4.0/) which permits unrestricted non-commercial use, distribution, and reproduction in any medium, provided the original work is properly cited. 

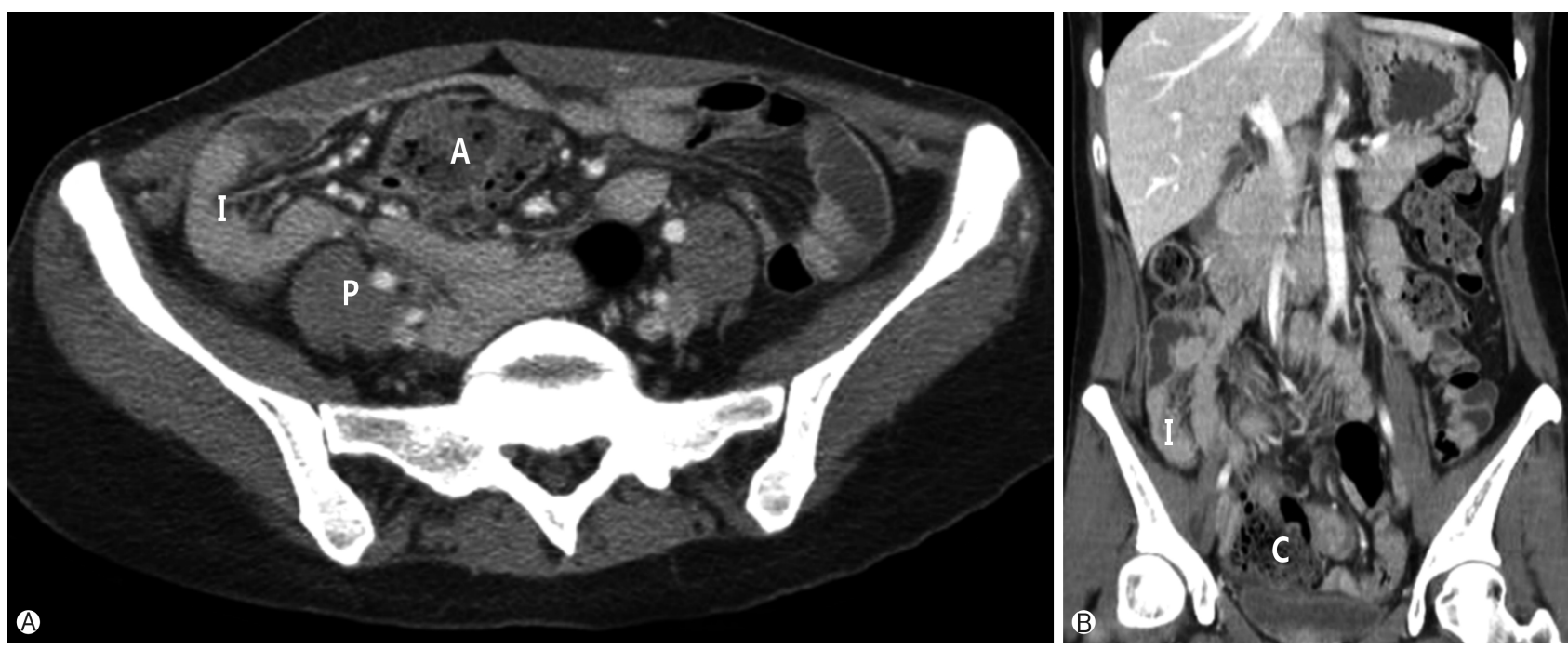

Fig. 1. Abdominopelvic computed tomography images. (A) The herniated ileum can be observed lateral to the ascending colon. (B) The cecum can be observed in the mid-pelvis. A, ascending colon; I, ileum; P, psoas muscle; C, cecum.
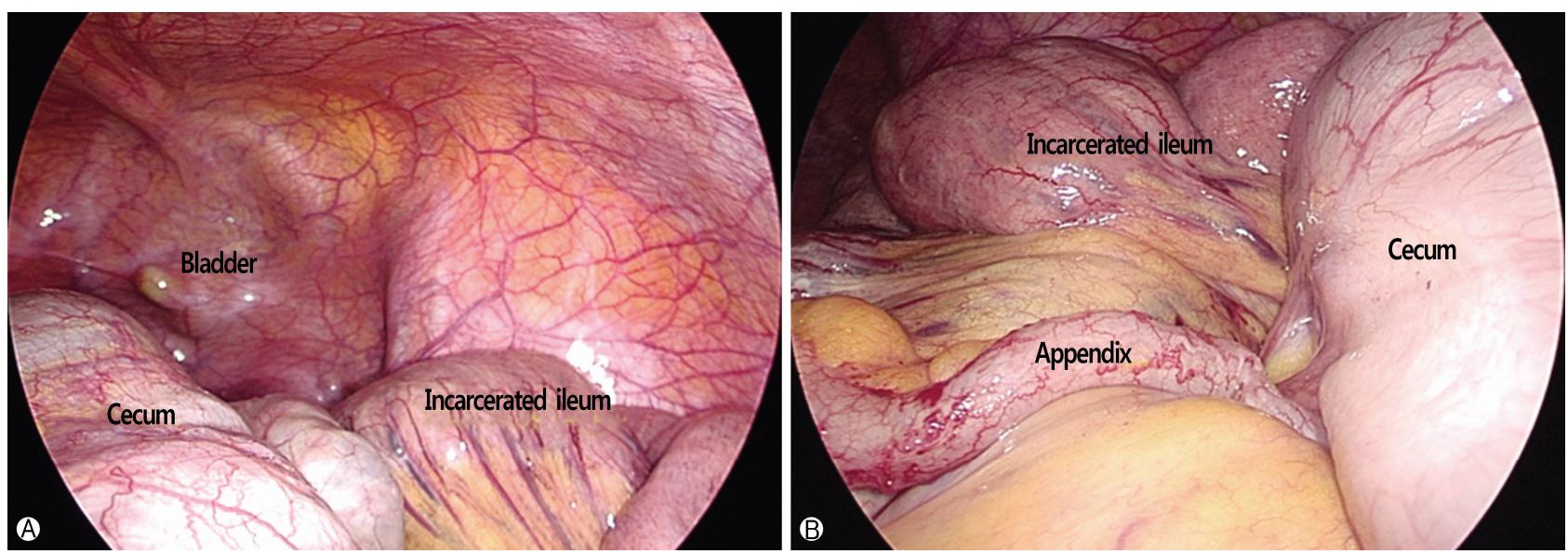

Fig. 2. Intraoperative laparoscopic images. (A) An atypical location of the cecum can be observed. (B) The incarcerated ileum is observed through the inferior ileocecal fossa.
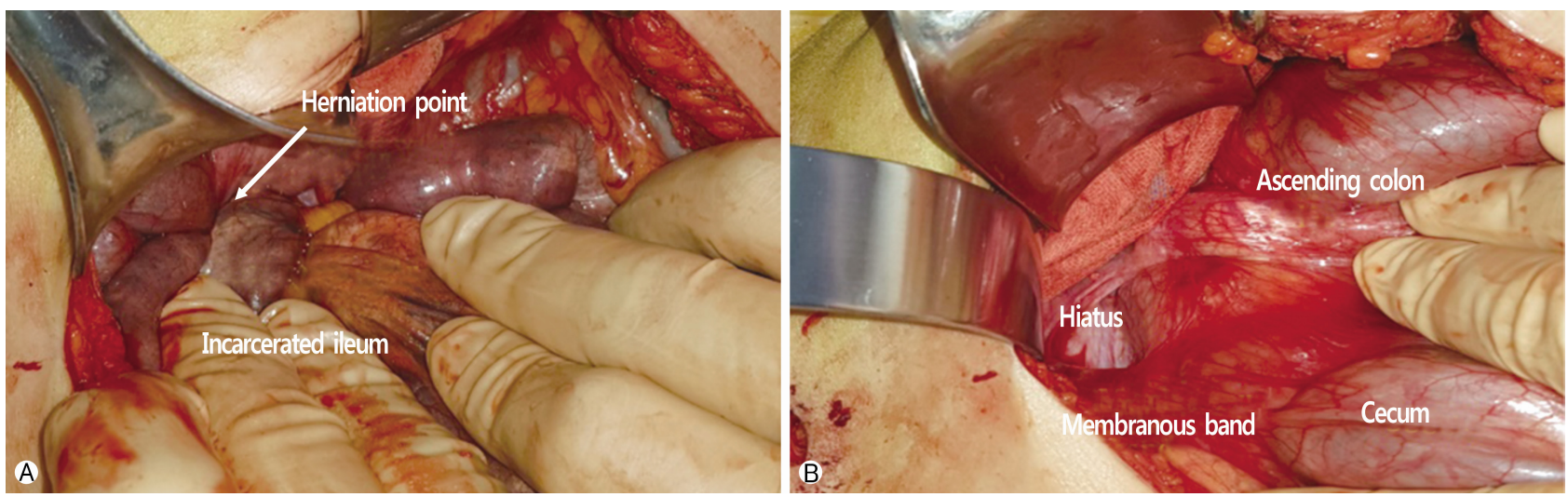

Fig. 3. Intraoperative images after conversion to open surgery. (A) The incarcerated ileum is observed through an inferior ileocecal fossa. (B) A membranous band, which formed an inferior ileocecal fossa can be identified, which was observed after manual reduction of the herniated ileum. 
ated ileum, the hernia orifice was noted as a form of the membranous band between the posterior cecal wall and the right colic gutter (Fig. 3). The membranous band was opened after manual reduction of the herniated ileum to prevent recurrence. Additionally, an appendectomy was performed. The patient verbalized relief of abdominal pain and was discharged on the 6th postoperative day without complication.

\section{DISCUSSION}

An internal hernia, which is defined as a bowel protrusion into a congenital or acquired visceral hiatus, is a rare cause of intestinal obstruction, accounting for $<6 \%$ of all cases of small bowel obstruction [3]. A pericecal hernia is a rare form of an internal hernia.

A pericecal hernia, also known as ileocolic, retrocecal, ileocecal, or paracecal hernia, has been reported in various incidences constituting $0.1-13 \%$ of all cases of internal hernias [4-6]. A hypothesis that could explain the mechanism associated with this type of hernia is abnormal intestinal rotation during embryonic development, which can cause an atypical location of the cecum and appendix and form pockets or recesses [3]. There are four possible peritoneal recesses located around the cecum: the superior, inferior ileocecal, retrocecal, and retro-appendicular fossa. In our case, the cecum was located in the mid-pelvis and as a result, a membrane which formed the retrocecal fossa caused herniation of the ileum [6,7].

To date, only one case of a pericecal hernia has been reported in Korea. Jang et al. [3] reported jejunal herniation through the cecal fossa with symptoms of bowel obstruction. In contrast, our patient demonstrated herniation of the distal ileum through the inferior ileocecal fossa. The clinical symptom observed in our patient was only vague abdominal pain without any signs of obstruction. Although our patient revealed a history of the previous laparotomy, no anatomical abnormality attributable to the previous laparotomy could be identified other than a few adhesion between the anterior peritoneum and the great omentum.

Contrast-enhanced CT is the best modality for the diagnosis of pericecal hernia. In this patient, the displaced cecum was observed with a small bowel loop located lateral to the anteromedial side of the cecum [4].

An emergency or urgent surgical procedure is the treatment recommended for a pericecal hernia because of the high risk of strangulation of the incarcerated bowel. Enterectomy of the strangulated bowel can be avoided if early diagnosis is possible and an urgency operation can be performed. Nishi et al. [7] have reported that $41 \%$ of the surgeries performed for the management of a pericecal hernia was performed without performing an enterectomy among the 51 cases that have been reported in Japan. This indicates that early diagnosis and prompt surgical intervention are important to ensure favorable prognosis.

Minimal invasive surgery such as laparoscopic surgery can be a useful surgical option for pericecal hernia. In this case, the patient has a history of laparotomy, and the operative scar was located in the midline of the upper abdomen. we tried to perform single-port laparoscopic surgery through the scar site for cosmetic purposes. It was therefore difficult to access the hernia site, which was located in the right low quadrant of the abdomen. Although single-port laparoscopic surgery failed in this patient because of mesenteric bleeding, we propose that laparoscopic surgery can be successfully performed in patients with a pericecal hernia.

In summary, a pericecal hernia is a rare form of an internal hernia, which can cause bowel incarceration. CT can diagnose this hernia, which requires prompt surgical reduction owing to the high risk of bowel strangulation.

\section{CONFLICT OF INTEREST}

No potential conflict of interest to this article was reported.

\section{ORCID}

Sung Il Kang, https://orcid.org/0000-0002-4751-5779

\section{REFERENCES}

1. Fu CY, Chang WC, Lu HE, Su CJ, Tan KH. Pericecal hernia of the inferior ileocecal recess: CT findings. Abdom Imaging 2007;32:81-3.

2. Lu HC, Wang J, Tsang YM, Tseng HS, Li YW. Pericecal hernia: a report of two cases and survey of the literature. Clin Radiol 2002; 57:855-8.

3. Jang EJ, Cho SH, Kim DD. A case of small bowel obstruction due to a paracecal hernia. J Korean Soc Coloproctol 2011;27: 41-3. 
4. Yeung KW, Hsiao CP. Evaluation of acquired pericecal hernia using computed tomography: a case report. J Radiol Sci 2013; 38:101-3.

5. Liu KL, Lin BR. Images in clinical medicine. Small-bowel obstruction due to an internal hernia. N Engl J Med 2007;356: 1152.
6. Rivkind AI, Shiloni E, Muggia-Sullam M, Weiss Y, Lax E, Freund HR. Paracecal hernia: a cause of intestinal obstruction. Dis Colon Rectum 1986;29:752-4.

7. Nishi T, Tanaka Y, Kure T. A case of pericecal hernia with a hernial orifice located on the lateral side of the cecum. Tokai J Exp Clin Med 2011;36:71-4. 